\title{
The Fine Structure of the Longitudinal Machine Impedance Observed with Single Bunches
}

\author{
T.Bohl, T.Linnecar, E.Shaposhnikova \\ CERN, SL Division, 1211 Geneva 23, Switzerland
}

\begin{abstract}
Using a beam spectrum measurement the detailed structure of the CERN SPS longitudinal machine impedance has been observed over a wide frequency range. Single high intensity proton bunches were injected into the machine with RF off. The spectrum of the unstable bunch modes, produced by the different resonant impedances in the ring, was measured during slow debunching. The maximum mode amplitude as a function of frequency was recorded for many injected bunches. The central frequency of each bunch mode is close to the resonant frequency of the impedance and the width depends upon the bunch length. Using sufficiently long bunches allowed the machine impedance to be seen in detail in the frequency range $100 \mathrm{MHz}$ to $4 \mathrm{GHz}$. The low frequency of this range is defined by the initial stable bunch spectrum, and the high frequency by the vacuum chamber cut-off frequency. The dominant peaks in the spectrum have been identified with different impedance sources in the machine - some previously unknown.
\end{abstract}

\section{INTRODUCTION}

When trying to understand or predict the behaviour of intense beams in particle accelerators, accurate knowledge of the machine coupling impedance is essential. The design of new accelerators profits from an estimation of the impedance by powerful numerical codes. Older machines which have not been able to benefit as much from these calculations usually have significantly larger and less well known impedance.

The SPS, built in the 70's, has a non-smooth vacuum chamber with many cross section changes around its circumference. The SPS has exceeded its initial design intensity many times over, but must now be upgraded to be the injector for the new LHC machine. One of the critical beam parameters for this mode of operation is the longitudinal emittance. The emittance at extraction at $450 \mathrm{GeV}$ must be kept between $0.5 \mathrm{eVs}$ and $1 \mathrm{eVs}$ with a preference from LHC for the lower value defined by dynamic aperture [1]. In addition if the emittance turns out to be higher than $0.6 \mathrm{eVs}$, clean injection into the LHC would require a new additional RF system in the SPS. The emittance at injection at $26 \mathrm{GeV}$ is foreseen as $0.35 \mathrm{eVs}$ which will increase to $\sim 0.5 \mathrm{eVs}$ after the matching process [2]. Consequently great care must be taken to control emittance blow up and therefore both single and coupled bunch instabilities during the cycle. Note that the beam of 243 bunches, intensity $10^{11}$ per bunch, filling $3 / 11$ of the machine circumference, combines the high single bunch intensities obtained during
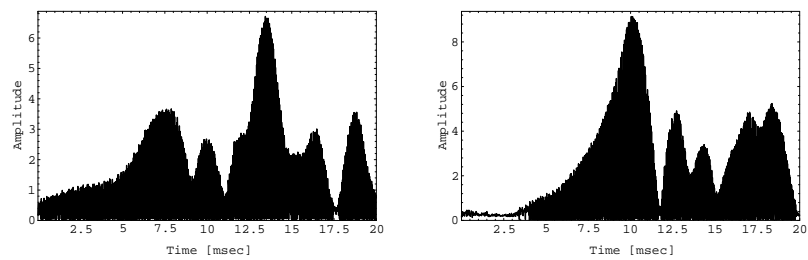

Figure 1: Examples of beam signals at $1.56 \mathrm{GHz}$

collider operation in the past, where the microwave instability was a limitation [3], with the large total intensities achieved in fixed target operation, where coupled bunch instabilities are seen.

The microwave instability, first observed in the SPS in 1976 [4], is not harmful for present operation, but it limits the single bunch intensity of the LHC beam. A new measurement technique [5], used last year and described below, has allowed us to determine the dominant sources of longitudinal impedance in the SPS responsible for this instability.

\section{SPECTRUM MEASUREMENT}

\subsection{Method of measurement and results}

The method was first found experimentally and then justified from linear theory and simulation. This is the "recipe" we followed in our measurements.

- Inject long single bunches above the instability threshold, (in our case at $26 \mathrm{GeV}$, bunch length $\tau=25-50 \mathrm{~ns}$, emittance $\varepsilon \sim 0.2-0.3 \mathrm{eVs}$ and intensity $N=1-5 \times 10^{10}$ particles per bunch). RF is off.

- Observe the signals growing after injection as a function of time, (wideband longitudinal pickup with spectrum analyser, bandwidth $3 \mathrm{MHz}$, used as a receiver). See examples in Fig.1. Record the maximum amplitude at each frequency, (centre frequency varied over from $100 \mathrm{MHz}$ to 4GHz).

- Take data at each frequency from a large number of bunches (at least 10).

- The bunch should debunch very slowly (length changes $<50 \%$ during the observation time $\sim 100 \mathrm{~ms}$ ).

The result of this method applied to the SPS in two different sets of measurements is shown in Fig.2. The fine structure of the beam spectrum, which was very reproducible, is clearly seen.

\subsection{Justification for method from linear theory}

We measured the peak mode amplitude of the instability and used simulation to confirm our interpretation of the re- 

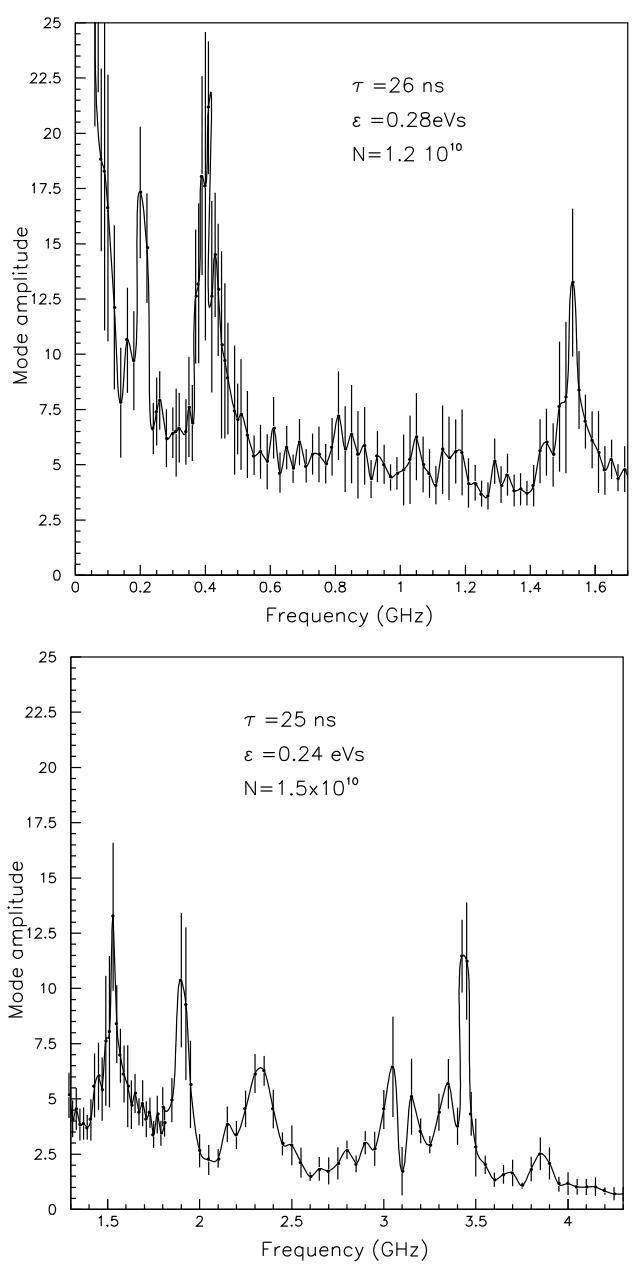

Figure 2: Structure from $0.1 \mathrm{GHz}$ to $4.0 \mathrm{GHz}$

sults for this non-linear regime. However it is possible to gain some information from the theory for the linear regime of a fast microwave instability. We assume: (a) the instability growth rate is much faster than the debunching time defined as the time when the bunch length increases by $\sqrt{2}$; (b) $\omega_{r} \tau \gg 1$, where $\omega_{r}$ is the centre frequency of the impedance, (many wavelengths cover the bunch); (c) the impedance bandwidth is less than the bunch spectrum bandwidth $\sim 1 / \tau$, (narrowband impedance regime), but not so low as to provoke strong coupled bunch phenomena. Then from linear theory the spectrum of the unstable bunch mode at azimuthal harmonic $n$ is,

$$
\Delta \rho_{n} \sim n \int_{-\pi}^{\pi} \rho_{0}(\theta) e^{-i\left(n-n_{r}\right) \theta} d \theta,
$$

where $\theta$ is the azimuthal coordinate, $\rho_{0}(\theta)$ is the bunch line density, $n_{r}=\omega_{r} / \omega_{0}$, and $\omega_{0}$ is the revolution frequency.

We see that the spectrum shape is given by the Fourier Transform (FT) of the stable bunch, shifted to the resonant frequency of the impedance. As an example Fig. 3 shows this solution in space and frequency domain for a parabolic bunch. Longer bunches have narrower spectral bandwidth giving finer resolution, (recipe item 1). This can be seen in reality in measurements on the SPS. In Fig.4 the bunch pro-
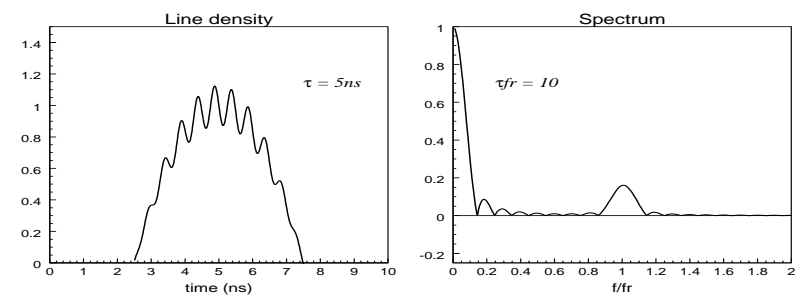

Figure 3: Profile and spectrum of unstable bunches

file measured every 100 turns from injection is presented in the first plot as a mountain range display. The unstable modes grow on the bunch and by taking the FT of each profile we see clearly in the second plot the increase of signals with definite width at each frequency corresponding to the different impedance sources. The projection of the spectra from Fig.4 averaged over many bunches would give similar results to Fig.2. Peaks appearing at different times explains why taking the maximum amplitude, (recipe item 2 ), reproduces many unstable modes. Note also the slow debunching.

The initial perturbation grows as $\Delta \rho_{n}(t) \propto e^{-i \Omega t}$, with instability for $\operatorname{Im} \Omega>0$. For a resonator with shunt impedance $R_{s h}$ and quality factor $Q$ the instability growth rate is given by

$$
\operatorname{Im} \Omega \simeq \omega_{r}\left(\frac{N e^{2} \omega_{0}|\eta|}{16 \pi E_{0}} \frac{R_{s h}}{Q}\right)^{\frac{1}{2}}
$$

where $\eta$ is the slip factor and $E_{0}$ is the synchronous energy.

In this approximation the growth rate is independent of the initial bunch distribution and proportional to $\left(R_{s h} / Q\right)^{1 / 2}$. The same formula can be applied when RF is on if the growth rate is much larger than the synchrotron frequency. It was first conjectured in [6] that for a single bunch instability due to a narrow band impedance, the instability threshold should depend on $R_{s h} / Q$ and average current, in contrast to the broad band impedance case $\left(1 / \tau \ll \omega_{r} / 2 Q\right)$ where $Z / n$ and peak current are important.

From Eq.2 we draw the important conclusion that although damping resonators (reducing $R_{s h}$ but $R_{s h} / Q=$ const) can always help cure coupled bunch instabilities, this does not apply to the single bunch case until the broadband impedance regime is reached.

\section{IMPEDANCE SOURCES}

Using the results of the measurements shown in Fig.2, it was possible to determine the sources of the dominant impedances in the SPS. The peaks at $200 \mathrm{MHz}$ and $800 \mathrm{MHz}$ are from the fundamental mode of the two travelling wave (TW) RF systems which have high $R_{s h} / Q$ and low Q ( 130). Small peaks from $0.4 \mathrm{GHz}$ to $1.4 \mathrm{GHz}$ can be identified with the HOM modes of the six RF systems in the SPS [7]. However the sources of peaks at $400 \mathrm{MHz}$ and above $1.4 \mathrm{GHz}$ were not obvious.

Frequencies above $1.4 \mathrm{GHz}$ suggest cavity-like devices with a radius $r$ of around $8 \mathrm{~cm}$. In the SPS there are about 

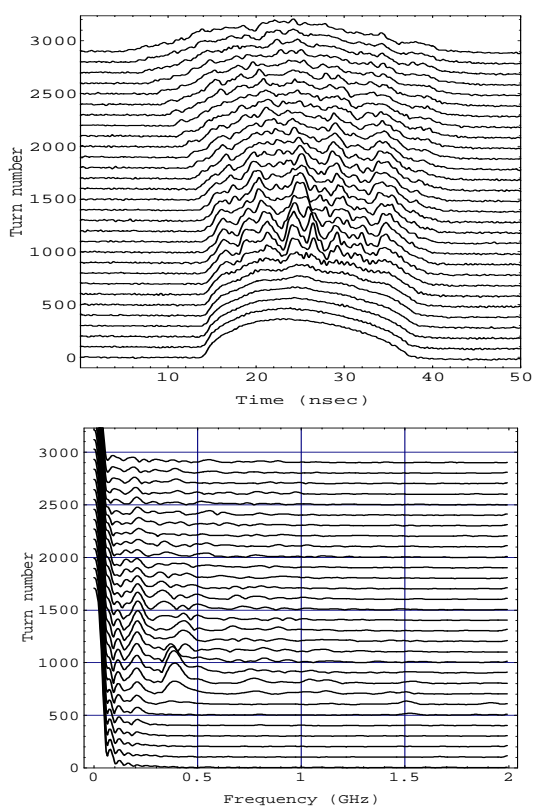

Figure 4: Fourier analysis of longitudinal bunch profiles.

800 pumping ports which fit this criterion and can explain all high frequency resonances. It is interesting that the simple formula for the resonant frequency for the $T M_{m n l}$ modes of a cylindrical cavity

$$
f_{r}=\frac{c}{2} \sqrt{\left(\frac{l}{z}\right)^{2}+\left(\frac{u_{m, n}}{\pi r}\right)^{2}}
$$

is in good agreement with the frequencies observed. Here $z$ is the cavity length, $l$ is the axial mode number and $u_{m, n}$ is the $n$-th root of equation $J_{m}(u)=0, J_{m}(x)$ being the Bessel function. For an axial longitudinal electric field, $T M_{0 n l}$ modes, $u_{01}=2.405$ and $u_{02}=5.52$.

The lowest frequencies of the $T M_{01 l}$ and $T M_{02 l}$ modes $(l=0,1,2 \ldots)$ from Eq. 3 for $r=8.2 \mathrm{~cm}$ and $z=24.0 \mathrm{~cm}$, are $1.4,1.53,1.88,2.34,2.87,3.2,3.42,3.7$ and 4.0GHz. They can all be seen in Fig. 2 at close frequencies. Simulation shows a frequency shift between $\omega_{r}$ and the mode centre frequency increasing with intensity. The code MAFIA was used [8] for a more precise 3-D geometrical representation. It also gives an estimation of $R_{s h} / Q$. The 1 st mode has low $R_{s h} / Q=3.9 \Omega$ (one cavity) and is hardly visible in the spectrum whereas the 2 nd has $27.8 \Omega$ and gives a large peak. These accidental cavities were always damped by ceramic resistors - laboratory measurements give a $Q \sim 70$. This $Q$ is quite low but due to the wide spectrum of the LHC bunches $(\tau<5 \mathrm{~ns})$ this case corresponds to the narrowband impedance regime. We believe that the pumping ports are the main cause of microwave instability in the SPS.

A search for the source of the unknown peak at $400 \mathrm{MHz}$ led finally to the identification of the magnetic extraction septa as the guilty elements.

\section{GROWTH RATE MEASUREMENTS}

The measured spectrum in Fig. 2 suggests the frequencies of the various sources. The amplitude of the peaks already gives some indication of $R_{s h} / Q$. For a further estimation we have tried to use growth rate measurements. Indeed, from Eq.2, $R_{s h} / Q \propto\left(\operatorname{Im} \Omega / \omega_{\mathrm{r}}\right)^{2}$. However these measurements are difficult for the following reasons. First the stable bunch spectrum moves with debunching. Second, all frequencies within the unstable bunch spectrum grow simultaneously. This means that sources close together can contaminate each other, see Fig.1 (left). This leads to a huge scatter in the measurements. However in some cases the signal is relatively clean, see Fig.1 (right). This signal, at $1.56 \mathrm{GHz}$, has an average growth time of $2 \mathrm{~ms}$ for $\mathrm{N}=4$ $\times 10^{10}$. This gives $R_{s h} / Q=11.5 \mathrm{k} \Omega$ which should be compared with the value of $21.7 \mathrm{k} \Omega$ given by MAFIA. We also used threshold measurements at given frequencies, observing the maximum amplitude as a function of intensity to estimate this impedance [9], and obtained the same results.

\section{CONCLUSIONS}

Our present SPS impedance model to describe single bunch phenomena consists of the 12 dominant resonant impedances whose frequencies were found from measurements with the beam. $R_{s h} / Q$ and $Q$ values were obtained from MAFIA calculations and laboratory measurements, respectively. These impedances correspond to four main sources: two TW RF systems, extraction septa and vacuum ports. Using this model for the LHC beam in the SPS suggests that the beam with nominal intensity, $10^{11}$ protons per bunch, will be at the limit of instability. Knowing the guilty elements in the machine measures will be taken to reduce their impedance in readiness for the SPS as LHC injector.

Finally we believe that this method of impedance measurement could be applied to other accelerators.

We thank R.Cappi, D.Manglunki and the CERN PS and SPS operations groups for their help. We are grateful to D.Boussard for useful discussions.

\section{REFERENCES}

[1] J.Gareyte, Proceedings of the LHC96 Workshop, Montreux, to be published in Particle Acc.

[2] R.Garoby, CERN PS/RF/Note 93-17, 1993.

[3] L.R.Evans, J.Gareyte, CERN SPS/85-15 (DI-MST), 1985.

[4] D.Boussard, G.Dome, T.Linnecar, A.Millich, IEEE Trans. on Nucl. Science, Vol.NS-24, No.3, 1977, p.1399.

[5] T.Bohl, T.Linnecar, E.Shaposhnikova, Phys. Rev. Lett., 21st April 1997, p.3109.

[6] K.Y.Ng, Proc. 1986 Summer Study on Phys. of SSC, 1986, p.590.

[7] T.Linnecar, E.Shaposhnikova, CERN SL-Note 96-49 (RF), 1996.

[8] W.Hofle, SL/Note 96-40 (RF), 1996.

[9] T.Linnecar, E.Shaposhnikova, Proceedings of the LHC96 Workshop, Montreux, to be published in Particle Acc. 